\title{
Capacity for development of primary care residency programs in Non-Teaching hospitals in New York State (NYS)
}

\author{
Fabio V. Lima ${ }^{1}$, Karen Goldsteen ${ }^{2}$, Raymond L. Goldsteen ${ }^{3}$, Laura Chiu ${ }^{4}$, Shamuel Yagudayev ${ }^{5}$, \\ Norman H. Edelman ${ }^{6, *}$
}

${ }^{1}$ Medical Student at Stony Brook University School of Medicine, and affiliate of, Department of Preventive Medicine and Program in Public Health, Stony Brook University, Stony Brook, New York.

${ }^{2}$ Associate Professor of the Master of Public Health Program, University of North Dakota School of Medicine and Health Sciences, Grand Forks, North Dakota

${ }^{3}$ Professor and Founding Director, Master of Public Health Program, University of North Dakota School of Medicine and Health Sciences, Grand Forks, North Dakota

${ }^{4}$ Medical Student, Albany Medical College, Albany, New York, and affiliate of, Department of Preventive Medicine and Program in Public Health, Stony Brook University, Stony Brook, New York

${ }^{5}$ Medical student, Ross University School of Medicine, Commonwealth of Dominica and affiliate of, Department of Preventive Medicine and Program in Public Health, Stony Brook University, Stony Brook, New York

${ }^{6}$ Professor of Preventive Medicine and Internal Medicine and core member of the Program in Public Health, Stony Brook University, Stony Brook, New York

\section{Email address:}

norman.edelman@stonybrook.edu (N. H. Edelman)

\section{To cite this article:}

Fabio V. Lima, Karen Goldsteen, Raymond L. Goldsteen, Laura Chiu, Shamuel Yagudayev, Norman H. Edelman. Capacity for Development of Primary Care Residency Programs in Non-Teaching Hospitals in New York State (NYS). American Journal of Health Research. Vol. 3, No. 1, 2015, pp. 13-17. doi: 10.11648/j.ajhr.20150301.13

\begin{abstract}
Purpose: The demand for physicians in the U.S., especially those practicing adult primary care, is accelerating and will inevitably require the expansion of residencies, despite current constraints on funding for graduate medical education (GME). A previous study showed little interest in or capacity for expansion of primary care residencies in current teaching hospitals in NYS. This study examines the interest in and capacity for establishing new programs among current non-teaching hospitals in that state. Design and Methods: Chief Executive Officers of eligible non-teaching hospitals in NYS were surveyed and asked if they had interest in establishing a residency program, what medical specialties they would choose assuming availability of additional funds, and what barriers there were to residency development. Results: Fourteen of 46 (30\%) NYS sites completed the survey. All but one was interested in establishing residencies; 85 percent would establish new programs in Emergency Medicine; 76 percent in Family Medicine; and 54 percent in Internal Medicine and/or Primary Care Internal Medicine. Virtually all cited significant concerns related to funding, faculty supply, and need for medical school affiliations. Conclusions: A minimum of 28 percent of non-teaching hospitals in NYS have a significant interest in establishing a GME program. If implemented this could increase training in Family Medicine by 40 percent and Internal Medicine by 11 percent. However, there are formidable financial and structural barriers to doing so. Enhanced support programs that go beyond lifting of the current GME cap will be necessary to increase the training of primary care physicians.
\end{abstract}

Keywords: Graduate Medical Education, Primary Health Care, Family Medicine, Medical Residency, Medical Workforce

\section{Introduction}

Projected changes in healthcare delivery have heightened attention to the need for more primary care physicians in the
U.S. The enactment of the Affordable Care Act (ACA) is projected to insure an additional 30 million or more Americans amidst an aging and growing population. Family physicians, internists, and pediatricians will likely play larger roles in coordinating the care of each patient. As a result, an 
estimated 45,000 more primary care physicians, especially those providing adult ambulatory care, are expected to be needed in the next decade. ${ }^{1,2}$

In the United States, medical residencies (Graduate Medical Education, GME) play the key role in determining the number and specialty mix of practicing physicians. Despite the increasing demand for medical services, Federal support for GME has changed little since Medicare support for resident education was "capped" in 1997 although there has been a modest amount of new funding for community based primary care residencies. ${ }^{3}$ We believe, however, that because of increasing demands, pressures for expansion of GME must eventually prevail, whatever the source of funding. To understand how the various interacting interests will shape the output of GME, particularly for primary care residencies, it is important to determine the level of interest in and capacity for expansion or establishment of resident training among current and potential teaching hospitals. In a previous study, we found that even if additional funding were provided at prevailing rates, current teaching hospitals were largely saturated with regard to the capacity to train additional internists and mostly uninterested in adding Family Medicine residency programs, despite considerable capacity to do so. ${ }^{4}$ Therefore, to understand whether there is additional capacity to train Family Medicine and other practitioners, we have done a similar study of current nonteaching hospitals. Both studies were done of New York State Institutions in order to have a manageable data base and because in some ways it is a laboratory for GME.

The present data show that, in contrast to current teaching hospitals, most of the responding non-teaching hospitals would sponsor or affiliate with Family Medicine residencies. Indeed if all respondents were successful we estimate that the output of Family Medicine practitioners in NYS could be increased by about 40 percent. We note in our analyses that, in order to increase the number of Family Medicine practitioners in the US programs are needed to provide support, financial and otherwise, to those current non- teaching hospitals which are interested in developing medical residencies.

\section{Design and Methods}

Our study consisted of a web-based survey throughout the year 2010 of Chief Executive Officers (CEO) at non-teaching hospitals (i.e., not American Council on Graduate Medical Education (ACGME) or American Osteopathic Association Sponsoring Institutions, nor receiving residents from sponsoring institutions) to determine the level of interest in and capacity for developing residency training programs. The list of non-teaching hospitals in NYS was obtained from the 2009 American Hospital Association (AHA) Guide. ${ }^{5}$ We identified all New York State hospitals which had more than 70 staffed beds and were no single specialty hospitals. For non-teaching hospitals that were part of a system, we chose to contact the system's CEO rather than each individual hospital CEO in the system. In total, there were 47 CEOs eligible to participate in the study.

The survey elicited information from the CEOs or their designees about the desire to sponsor first certification residency programs if external funding became available, that is, to become a sponsoring institution under the current funding formula or to affiliate with an existing sponsoring Institution. When desire to sponsor or affiliate was expressed, we determined which specialties were of interest from a list of first certification ("core") specialties. Table 1 lists these residencies. Furthermore, we asked all CEOs "Which of the following factors affect your hospital/medical center's decision not to sponsor resident programs at this point?" The choices provided are listed with their responses in Table 2. Since all surveyed institutions were hospitals or hospital systems, we also determined bed capacity for each using the 2009 AHA Guide. This project was approved by the Institutional Review Board of Stony Brook University. For statistical analyses we utilized Microsoft Excel 2010 (Microsoft Corp., Redmond, WA).

Table 1. Interest of Non-Teaching Hospitals in Sponsoring New or Affiliating with Existing Residency Programs ( $n=14)$

\begin{tabular}{llll}
\hline Residency Program & Would Sponsor & Residency Program \\
\hline Emergency Medicine & 11 & Emergency Medicine \\
Internal Medicine & 6 & Family Medicine & Would Affiliate \\
Internal Medicine, Primary Care & 5 & Internal Medicine & 11 \\
Anesthesiology & 4 & Internal Medicine, Primary Care \\
Psychiatry & 3 & Psychiatry & 7 \\
Family Medicine & 2 & General Surgery \\
Obstetrics and Gynecology & 2 & Anesthesiology \\
Urology & 2 & Obstetrics and Gynecology \\
Neurology & 1 & Orthopedic Surgery \\
Orthopedic Surgery & 1 & Neurology \\
Otolaryngology & 1 & Urology \\
Pathology & 1 & Pathology \\
Pediatrics & 1 & Pediatrics \\
Diagnostic Radiology & 1 & Diagnostic Radiology \\
General Surgery & 1 & Otolaryngology & 5 \\
& & Physical Medicine and Rehabilitation \\
\hline
\end{tabular}

Note: There was no interest in sponsoring or affiliating with other first certification residencies. 


\section{Results}

Of the 46 eligible non-teaching hospital sites, 14 agreed to participate, resulting in a response rate of 30 percent. All but one of those responding to the survey expressed interest in establishing GME programs, either by sponsoring their own programs or affiliating with established programs. There was no clear difference between responders and non-responders with regard to bed capacity.

Table 1 displays the programs that non-teaching hospitals had an interest in either sponsoring or affiliating with. With the exception of Emergency Medicine, there was considerably more interest in affiliation than sponsorship. The specialty of greatest interest was Emergency Medicine, with approximately 85 percent citing interest in sponsoring new residency programs in Emergency Medicine. If funding were available, 76 percent of respondents would develop new programs in Family Medicine, mostly by affiliation, while 54 percent would develop new programs in Internal Medicine and/or Primary Care Internal Medicine. For surgery and surgical subspecialties, there was considerably more interest in affiliation than sponsorship.

Table 2 lists the barriers to development of residency programs that were specified by the non-teaching hospitals. The great majority cited the need for funding beyond current reimbursement rates, insufficient faculty, lack of a medical school affiliation, and lack of space and other infrastructure issues.

Table 2. Reported Barriers to Adding New Programs among Non-Teaching Hospitals

\begin{tabular}{llll}
\hline \multirow{2}{*}{ Financial } & & Would Sponsor (n=13) & Would not sponsor (n=1) \\
& Insufficient additional funding sources & 10 & 0 \\
Resources & Insufficient reimbursement rates for residents & 8 & 0 \\
& Insufficient number of full time faculty & 9 & 1 \\
& Lack of medical school affiliation & 9 & 0 \\
& Insufficient number of qualified voluntary faculty & 8 & 1 \\
Infrastructure & Insufficient number of administrators & 4 & 0 \\
\multirow{4}{*}{ Administrative } & Insufficient space & 8 & 1 \\
& Other infrastructure concerns & 8 & 1 \\
\multirow{5}{*}{ Other } & Insufficient interest from medical staff & 2 & 1 \\
& Insufficient interest from governing board & 1 & 1 \\
& Insufficient interest from administrative staff & 0 & 0 \\
& Perceived inability to recruit residents of preferred quality & 3 & 1 \\
& Residency Review Committee (RRC) approval & 2 & 1 \\
\hline
\end{tabular}

We estimated how many entry-level adult primary care residency positions might be added through new programs if additional funding could be provided and other barriers overcome. To calculate this impact, we assumed that all Family Medicine residents and one third of Internal Medicine residents ${ }^{6}$ will go on to become providers of adult primary care. We assumed that the number of entry-level position in new programs, at maturity, would equal that of the median of entry-level positions in current programs in hospitals of similar bed capacity. Furthermore, it was also clear from Table 1 that the "affiliation" category subsumes the "sponsor" category. We thus considered the "affiliation" category to reflect the total interest in adding new residency programs. Finally, we made the conservative assumption that the responding hospitals represented all of those interested in developing GME among non-teaching hospitals. That is, we assumed that there was no interest in developing GME among the non-teaching hospitals that did not respond to the survey.

Using this approach, the data suggest that if the responding non-teaching hospitals in New York State were able to sponsor or affiliate as desired, the increase in yearly output of adult primary care physicians could be 136 (80 Family Medicine and 56 Internal Medicine). To provide a point of reference, in 2009 in New York State, there were 194 entry level positions in Family Medicine and 1554 entry level positions in Internal Medicine. ${ }^{7}$ Thus, there is at least sufficient interest in new programs among non-teaching hospitals to increase the output of Family Medicine physicians by about 40 percent and increase primary care Internal Medicine by 11 percent in New York State. From this analysis, we note that that providing GME funding at current rates and other support to current non-teaching hospitals could make a significant contribution to the training of practitioners of adult primary care medicine, particularly in Family Medicine, if their perceived barriers could be overcome. In addition, training in Emergency Medicine would be substantially increased.

\section{Discussion}

It is obvious from examination of the data that we cannot consider the responses to represent a representative sample of all non-teaching hospitals in NYS. Rather, since all but one of the respondents were interested in establishing a GME program, we take the view that the respondents represent, at a minimum, the number of institutions interested in doing so. This is a conservative viewpoint as there may indeed be more interested institutions. However, the response rate was great enough for us to conclude that if all responding hospitals were able to mount the programs they were interested in, the output of Family Physicians in NYS could be increased by a 
very significant $40 \%$. Another conservative assumption we have made is that the total capacity for expansion of GME in this cohort is represented by the desire to affiliate. That is we assumed those who expressed interest in sponsorship also expressed interest in affiliation and we did not sum the two columns in table one to determine total interest.

Accordingly, the findings of this study are that at least twenty eight percent of current non-teaching hospitals in NYS are interested in developing GME programs. Similar to the current teaching hospitals the greatest interest was in Emergency Medicine. This is not surprising given the increasingly important role Emergency Medicine departments play as the "front door" of all hospitals which have them. ${ }^{8}$ However, very much unlike the current teaching hospitals, 70 percent would also develop programs in Family Medicine. In addition there was a significant interest in primary care Internal Medicine. This finding is consistent with other observations if we consider that non-teaching hospitals are, in general, smaller than teaching hospitals. Thus, Chen et. al. have observed that teaching hospitals with fewer residents devote a greater percentage of their GME to training in primary care specialties than larger programs. ${ }^{9}$ In addition, we have recently observed that, nationwide, hospitals with small GME programs (fewer than 6 residencies) value their Family Medicine residencies much more from a financial and operational point of view than do institutions with larger programs. ${ }^{10}$ These observations are consistent with the more important role of primary and secondary care in smaller hospitals. In this setting, primary care residents not only care for hospitalized patients but function in primary care outpatient settings which are an important source of admissions for these hospitals.

Thus, important inroads in the national need for additional practitioners could be made if GME programs could be established in current non-teaching hospitals. However the findings of the study point out important limitations. Except for Emergency Medicine, the desire to affiliate was greater than the desire to sponsor new programs. This undoubtedly is related to the perceived barriers to establishing GME programs shown in table two. Although financial issues lead the list, lack of qualified faculty and lack of a medical school affiliation follow closely. Thus, it seems likely that for current non-teaching hospitals to become viable resources for expansion of GME they mostly will have to be incorporated into or at least affiliated with current GME programs rather than develop new programs on their own.

One argument against expansion of residencies in the primary care and other "less competitive" specialties has been a relative lack in interest in these specialties by graduates of U.S. medical schools. However, there always have been sufficient international medical graduates to fill or almost fill these positions and there is no evidence that these physicians perform less well in practice than graduates of U.S. medical schools. In addition, the ongoing expansion of undergraduate medical education in the U.S. is so great that it has been predicted that by the end of this decade there will be almost as many graduates as first year residency positions; thereby making the "less competitive" residencies more likely to be filled by U.S. graduates. ${ }^{11}$

A potential limitation of this study relates to sampling. As discussed above we do not believe that the respondents were a representative sample but rather that they represent a minimum number of non-teaching hospitals which are interested in mounting residency training programs. Additionally, New York is not a representative state with regard to GME. However, it is difficult to believe that Family Medicine and primary care Internal Medicine would be of less interest in other states compared to this specialty and sub-specialty oriented state. We also note that the study was done in 2010, a few years prior to publication of this report. However, there have been no substantive changes in GME in NYS since then, nor have there been any significant changes in alignments of non-teaching hospitals.

\section{Conclusions}

In conclusion, the present data show that there is sufficient interest in developing GME programs by current nonteaching hospitals to result in a significant increase in physician output in specialties currently thought to be of importance such as Family Medicine. However, materialization of this interest into actual programs will probably require both enhanced financial support as well as collaboration with established teaching entities such as academic health centers. Finally, because it seems unlikely that new governmental funds will be made available in the near future, urgently needed primary care residency expansion may require the redistribution of funds from other parts of GME. Redistributions have been suggested over the years and, not surprisingly, they have met considerable resistance. However there now seems to be stronger support for doing so in view of the recent report on GME by the Institute of Medicine ${ }^{12}$ which endorses reforms that would be favorable to smaller teaching hospitals and those which focus on current needs in physician education.

\section{Acknowledgments}

The authors would like to acknowledge the substantial contributions to this research and extend a special thanks to Fredrick Shiavone, MD, Associate Dean for GME at Stony Brook University for his input.

\section{List of Abbreviations}

$\begin{array}{ll}\text { AAMC } & \text { Association of American Medical Colleges } \\ \text { ACA } & \text { Affordable Care Act } \\ \text { ACGME } & \begin{array}{l}\text { American Council on Graduate Medical } \\ \text { Education } \\ \text { AMA }\end{array} \\ \text { American Medical Association } \\ \text { CEO } & \text { Chief Executive Officer } \\ \text { GME } & \text { Graduate Medical Education }\end{array}$




\section{References}

[1] Dorsey ER, Nicholson S, Frist WH. Commentary: improving the supply and distribution of primary care physicians. Acad Med. 2011; 86(5): 541-43.

[2] Association of American Medical Colleges. Physician shortages to worsen without increases in residency training [Internet]. Washington D.C.: Association of American Medical Colleges; 2010 [cited 2014 August 12]. Available from: https://www.aamc.org/download/150612/data/mdshortage.pdf.)

[3] Edelman N, Romesier J. Financing of graduate medical education. In: Johns MM, Chair. Ensuring an effective physician workforce for America: recommendations for an accountable graduate medical education system. Atlanta: Josiah Macy Jr. Foundation; 2010 October. p. 149-68.

[4] Edelman NH, Goldsteen RL, Goldsteen K, Yagudayev S, Lima F, Chiu L. Institutions with accredited residencies in New York State with an interest in developing new residencies or expanding existing ones. Acad Med. 2013; 88(9): 1287-92.

[5] Health Forum. AHA Guide to the Health Care Field, 2009 Edition. 1st ed. Washington, D.C.: American Hospital Association; 2008. 1100 p.

[6] Garibaldi RA, Popkave C, Bylsma W. Career plans for trainees in internal medicine residency programs. Acad Med. 2005; 80: 507-12.

[7] Smart DR. Physician characteristics and distributions in the US, 2011 Edition. 1st ed. Chicago: American Medical Association Press; 2010. 500 p.

[8] Schuur JD, Venkatesh AK. The growing role of emergency departments in hospital admissions. N Engl J Med. 2012; 367: 391-93.

[9] Chen C, Petterson S, Phillips RL, Mullan F, Bazemore A, O'Donnell SD. Toward graduate medical education (GME) accountability: measuring the outcomes of GME institutions. Acad Med. September 2013; 88(9):1267-80.

[10] Riaz MP, Palermo T, Yen M, Edelman, N H. Potential Responses of Sponsoring Institutions to Reduction of Medicare Support for Graduate Medical Education [GME]. Acad Med. 2014; Forthcoming.

[11] Jolly P, Erickson C, Garrison G. US graduate medical education and physician specialty choice. Acad Med. 2013; 88(4): 468-74

[12] J.Eden, D. Berwick, G.W. Wilensky. Graduate medical education that meets the nation's needs [Internet]. Washington, DC.: Institute of Medicine, Committee on the Governance and Financing of Graduate Medical Education; 2014 [cited 2014 November 16]. Available from: http://www.iom.edu/ /media/Files/Report\%20Files/2014/GM E/GME-RB.pdf 